\title{
Complexity of global semianalytic sets in a real analytic manifold of dimension 2
}

\author{
By A. Díaz-Cano and C. Andradas at Madrid
}

\begin{abstract}
Let $X \subset \mathbb{R}^{n}$ be a real analytic manifold of dimension 2. We study the stability index of $X, s(X)$, that is the smallest integer $s$ such that any basic open subset of $X$ can be written using $s$ global analytic functions. We show that $s(X)=2$ as it happens in the semialgebraic case. Also, we prove that the Hörmander-Łojasiewicz inequality and the Finiteness Theorem hold true in this context. Finally, we compute the stability index for basic closed subsets, $\bar{s}$, and the invariants $t$ and $\bar{t}$ for the number of unions of open (resp. closed) basic sets required to describe any open (resp. closed) global semianalytic set.
\end{abstract}

\section{Introduction}

In this paper we study the complexity of systems of inequalities in a paracompact real analytic manifold of dimension 2, namely, how to describe global semianalytic sets as economically as possible, i.e., using the minimum number of global analytic functions in their description.

The problem was settled by Bröcker at the beginning of the $80 \mathrm{~s}$, who introduced the invariants $s($ resp. $\bar{s})$ for the number of inequalities required to describe open (resp. closed) basic sets and $t$ (resp. $\bar{t}$ ) for the number of required unions of open (resp. closed) basic pieces. Bröcker and Scheiderer found, see [Brö] and [Sch], that if $V$ is an affine algebraic variety of dimension $n$ over a real closed field $R$ then $s(V)=n$ and $\bar{s}(V)=\frac{1}{2} n(n+1)$. Also, Bröcker gave bounds for the invariants $t(V)$ and $\bar{t}(V)$ which depend only on the dimension of $V$, cf. [Brö]. After that, Andradas, Bröcker and Ruiz found the same results for compact real analytic manifolds, see [An-Brö-Rz1] and [An-Brö-Rz2]. The same authors found $s\left(X_{0}\right)=n$ for a real analytic set germ $X_{0}$ of dimension $n$ and they also gave bounds for the invariants $\bar{s}, t$ and $\bar{t}$. Finally, the exact value of $\bar{s}\left(X_{0}\right)$ in arbitrary dimension and that of $t\left(X_{0}\right)$ in dimension 2 has been determined by Andradas and Díaz-Cano, cf. [DC1], [DC2] and [DC-An].

However, very little is known in the case of a global non-compact analytic set $X$, where the real spectrum does not behave as well as in the previous cases. The only known result was that if $X$ is a non-compact analytic manifold of dimension $1, s=\bar{s}=t=\bar{t}=1$, cf. [An-Be]. 
Here we study the invariants $s, \bar{s}, t$ and $\bar{t}$ in case $X$ is a two dimensional paracompact real connected analytic manifold and in our way to do that we compute also the values of $s$, $\bar{s}, t$ and $\bar{t}$ for singular global analytic curves, which turn out to be also 1 . Our approach to the problem is to exploit the interplay between orderings of the field of meromorphic functions on $X$ and global semianalytic sets given by the Artin-Lang property for two dimensional paracompact real connected analytic manifolds shown in [Ca1]. For the manipulation of the orderings we attach to each ordering of the field of meromorphic functions on $X$ an ultrafilter of global closed semianalytic sets. This method has already been used in $[\mathrm{An}-\mathrm{Be}],[\mathrm{Ca} 1],[\mathrm{Ca} 2]$ and $[\mathrm{Jw}]$.

We start by showing that $s(X)=2$. After that, Hörmander-Łojasiewicz inequality is found to be valid in this context. Using it we show that $\bar{s}(X)=t(X)=3$ and $\bar{t}(X)=2$ by means of the pasting lemmas proved in $\S 3$.

We want to thank the referee for his useful comments.

\section{Preliminaries}

Let $X$ be a paracompact real connected analytic manifold of dimension 2 . We denote by $\mathcal{O}(X)$ the ring of global analytic functions on $X$ and by $\mathscr{K}(X)$ (or simply $\mathscr{K}$ if $X$ is clear from the context) its quotient field. By Grauert's theorem, when needed we will assume that $X$ is a submanifold of $\mathbb{R}^{n}$ and $\mathcal{O}(X)=\frac{\mathcal{O}\left(\mathbb{R}^{n}\right)}{I}$ where $I$ is the ideal of analytic functions on $\mathbb{R}^{n}$ which vanish on $X$. We denote by $\mathcal{O}_{b}(X)$ the ring of bounded analytic functions on $X$.

A subset $S \subset X$ is called global semianalytic if it can be written in the form

$$
S=\bigcup_{i=1}^{p}\left\{x \in X \mid f_{i}(x)=0, g_{i 1}(x)>0, \ldots, g_{i j_{i}}(x)>0\right\}
$$

where $f_{i}, g_{i j} \in \mathcal{O}(X)$. The boundary of $S$ will be denoted by $\operatorname{Bd} S$. In the few occasions that the Zariski topology is used we will add the index $Z$ in the notations.

A subset of $X$ of the form $\{x \in X \mid f(x)>0\}$ (resp. $\{x \in X \mid f(x) \geqq 0\}$ ) with $f \in \mathcal{O}(X)$ is called principal open (resp. closed). A finite intersection of principal open (resp. closed) sets is called basic open (resp. closed). We denote by $s(X)$ (resp. $\bar{s}(X))$ the smallest integer such that every basic open (resp. closed) set is an intersection of at most $s(X)$ (resp. $\bar{s}(X))$ principal open (resp. closed) sets. If no such integer exists then $s(X):=\infty$ (resp. $\bar{s}(X):=\infty)$. The invariant $s(X)$ is called the stability index of $X$.

We also define the invariant $t(X)$ (resp. $\bar{t}(X)$ ) as the smallest integer such that every set $S$ which is a finite union of basic open (resp. closed) sets can be written as a union of at most $t(X)$ (resp. $\bar{t}(X))$ basic open (resp. closed) sets. As before $t(X):=\infty$ (resp. $\bar{t}(X):=\infty)$ if no such integer exists.

To any ordering $\beta$ of $\mathscr{K}$, we will associate the ring $W_{\beta}$, defined as the convex hull of $\mathbb{R}$ in $\mathscr{K}$ with respect to $\beta$, that is, 


$$
W_{\beta}:=\left\{f \in \mathscr{K} \mid-r<_{\beta} f<_{\beta} r \text {, for some } r \in \mathbb{R}^{+}\right\} .
$$

$W_{\beta}$ is the minimal $\beta$-convex valuation ring of $\mathscr{K}$, has residue field $\mathbb{R}$ and its maximal ideal, denoted by $\mathfrak{n}_{\beta}$, is made up from the elements of $\mathscr{K}$ which are infinitesimals with respect to $\beta$, that is,

$$
\mathrm{n}_{\beta}:=\left\{f \in \mathscr{K} \mid-r<_{\beta} f<_{\beta} r, \forall r \in \mathbb{R}^{+}\right\} .
$$

We denote by $U_{\beta}$ the set of units of $W_{\beta}$, that is, $U_{\beta}=W_{\beta} \backslash n_{\beta} ; \Gamma_{\beta}$ and $\omega_{\beta}$ stand respectively for the value group and the associated valuation of $W_{\beta}$. We have $\mathcal{O}_{b}(X) \subset W_{\beta}$ and the center of $W_{\beta}$ in $\mathcal{O}_{b}(X), \mathfrak{m}_{\beta}:=\mathfrak{n}_{\beta} \cap \mathcal{O}_{b}(X)$, is a maximal ideal in $\mathcal{O}_{b}(X)$ since the residue field of $W_{\beta}$ is $\mathbb{R}$.

We shall denote by $\mathscr{C}$ the family of closed global semianalytic subsets of $X$. Since $\mathscr{C}$ is closed under finite unions and intersections, it makes sense to consider filters in $\mathscr{C}$. In particular, given the ordering $\beta$ of $\mathscr{K}$, the family

$$
\mathscr{U}_{\beta}:=\left\{Y \in \mathscr{C} \mid Y \cap f^{-1}[-1,1] \neq \emptyset, \forall f \in \mathfrak{m}_{\beta}\right\}
$$

is a filter of sets of $\mathscr{C}$ which is maximal and will be called the ultrafilter attached to $\beta$. It can be checked that $\mathscr{U}_{\beta}$ defines indeed a unique point in the Stone-Cêch compactification of $X$ (see $[\mathrm{Jw}])$.

The usefulness of the filter $\mathscr{U}_{\beta}$ is given by the following important

Lemma 1.1. Let $f \in \mathcal{O}_{b}(X)$ and suppose that $\left.f\right|_{Y}>0$ for some $Y \in \mathscr{U}_{\beta}$. Then $f \in \beta$, and for each $n \in \mathbb{N}$ there exist $u, g \in \mathcal{O}_{b}(X)$ such that $u \in U_{\beta},\left.u\right|_{Y}>0$ and $u f=g^{n}$.

Proof. See [Ca2], Lemma 2 or [An-Brö-Rz2].

By $\beta$ we shall denote not only the ordering of $\mathscr{K}$ but also the cone of positive functions defined by it, so $f \in \beta$ means $f>_{\beta} 0$.

Remark 1.2. If the zero set of $f \in \mathcal{O}(X)$ is not in $\mathscr{U}_{\beta}$ then for each $n \in \mathbb{N}$ there exist $u, g \in \mathscr{K}$ with $u \in U_{\beta}$ such that $u f=g^{n}$. Indeed, since $\mathscr{Z}(f) \notin \mathscr{U}_{\beta}$ and $\mathscr{U}_{\beta}$ is an ultrafilter then there must exist $Y \in \mathscr{U}_{\beta}$ such that $\mathscr{Z}(f) \cap Y=\emptyset$. Thus we can write $Y=Y_{1} \cup Y_{2}$ with $Y_{1}=\{x \in Y \mid f(x)>0\}$ and $Y_{2}=\{x \in Y \mid f(x)<0\}$. Notice that $Y_{1}$ and $Y_{2}$ are closed sets so one of them must be in $\mathscr{U}_{\beta}$. Suppose $Y_{1} \in \mathscr{U}_{\beta}$ (otherwise we change $f$ by $-f$ ), that is, $\left.f\right|_{Y_{1}}>0$. Thus by Lemma 1.1 we have $u f=g^{n}$ for some $u \in U_{\beta}, g \in \mathscr{K}$.

We finish this section by recalling that for any field $K$ its stability index $s(K)$ (i.e. the stability index of its space of orderings $\operatorname{Spec}_{r} K$ ) is given by the so called Stability Formula due to L. Bröcker, cf. [An-Brö-Rz2], Corollary V.1.6:

$$
s(K)=\sup \left\{s \mid \text { there is a fan } F \subset \operatorname{Spec}_{r} A \text { with } \# F=2^{s}\right\},
$$

\section{Generic stability index}

In this section we compute the stability index of the field $\mathscr{K}$ of meromorphic functions on $X$. By the stability formula above, we have to determine the size of the fans in 
$\operatorname{Spec}_{r} \mathscr{K}$. One special type of fans are those made up from the orderings of $\operatorname{Spec}_{r} \mathscr{K}$ with the same minimal convex valuation ring $W$. We will call $F_{W}$ to these fans, that is,

$$
F_{W}:=\left\{\alpha \in \operatorname{Spec}_{r} \mathscr{K} \mid W_{\alpha}=W\right\} .
$$

The number of elements of these fans can be computed by the formula $\# F_{W}=\#\left(\frac{\Gamma_{W}}{2 \Gamma_{W}}\right)$. Of course, not all fans are of this type, but every fan in $\operatorname{Spec}_{r} \mathscr{K}$ is contained in the union of two fans of this type, cf. [Ma], 1.3, 3.1 and 3.5. Thus, computing the number of elements of the fans $F_{W}$ will bound the generic stability index $s(\mathscr{K})$.

We classify the orderings of $\operatorname{Spec}_{r} \mathscr{K}$ in three types:

A) Orderings $\beta \in \operatorname{Spec}_{r} \mathscr{K}$ whose attached ultrafilter $\mathscr{U}_{\beta}$ does not contain any proper analytic set. We will say that $\mathscr{U}_{\beta}$ has dimension 2 for it is the dimension of all sets in $\mathscr{U}_{\beta}$.

B) Orderings $\beta \in \operatorname{Spec}_{r} \mathscr{K}$ whose attached ultrafilter $\mathscr{U}_{\beta}$ contains proper analytic sets but no discrete sets. In this case we will say that $\mathscr{U}_{\beta}$ has dimension 1 for this is the minimum of the dimensions of the sets in $\mathscr{U}_{\beta}$.

C) Orderings $\beta \in \operatorname{Spec}_{r} \mathscr{K}$ whose attached ultrafilter $\mathscr{U}_{\beta}$ contains a discrete set. We will say that $\mathscr{U}_{\beta}$ has dimension 0 .

We start by counting the elements of $F_{\beta}$ in case $\mathscr{U}_{\beta}$ has dimension 2.

Proposition 2.1. Let $\beta \in \operatorname{Spec}_{r} \mathscr{K}$ and suppose that $\operatorname{dim} \mathscr{U}_{\beta}=2$. Then $\beta$ is the only ordering with that ultrafilter $\mathscr{U}_{\beta}$ and $\Gamma_{\beta}$ is divisible. In particular, $\frac{\Gamma_{\beta}}{2 \Gamma_{\beta}}=\{0\}$ and $\# F_{W_{\beta}}=1$.

Proof. Let $f \in \mathcal{O}_{b}(X)$. Since $\operatorname{dim} \mathscr{U}_{\beta}=2$ we must have $\mathscr{Z}(f) \notin \mathscr{U}_{\beta}$ so by Remark 1.2 we can write $u f=g^{n}$ for some $u \in U_{\beta}$. This implies that $\omega(f)=n \omega(g) \in n \Gamma_{\beta}$. In general, each $f \in \mathscr{K}$ can be expressed as a quotient of bounded analytic functions, so $\omega(f) \in n \Gamma_{\beta}$, $\forall f \in \mathscr{K}$. Thus, $\Gamma_{\beta}$ is divisible and $\# F_{\beta}=\#\left(\frac{\Gamma_{\beta}}{2 \Gamma_{\beta}}\right)=1$.

Moreover this shows that the ordering $\beta$ can be characterized as follows: If $f \in \mathcal{O}_{b}(X)$, then $f>_{\beta} 0$ if and only if there is $Y \in \mathscr{U}_{\beta}$ such that $\left.f\right|_{Y}>0$. In conclusion, if $\operatorname{dim} \mathscr{U}_{\beta}=2$ then the ultrafilter $\mathscr{U}_{\beta}$ defines uniquely the ordering $\beta$.

For the second type of orderings we need the following result, cf. [Ca1], Proposition 3.3. We recall that a function $f$ changes sign at $x$ if both sets $\{y \in X \mid f(y)>0\}$ and $\{y \in X \mid f(y)<0\}$ induce not empty set germs at $x$. We denote by $\mathscr{Z}(f)^{\mathrm{c}}$ the set of points where $f$ changes sign.

Proposition 2.2. Let $f \in \mathcal{O}(X)$. There exists a unique function (up to units) $f^{\prime} \in \mathcal{O}(X)$ such that:

a) $f=f^{\prime} \sum_{1}^{r} A_{i}^{2}$, with $A_{i} \in \mathcal{O}(X)$. In particular, $f$ changes sign iff $f^{\prime}$ does. 
b) $\mathscr{Z}\left(f^{\prime}\right)=\mathscr{Z}(f)^{\mathrm{c}}$.

c) $\forall x \in \mathscr{Z}\left(f^{\prime}\right), \mathscr{J}\left(\mathscr{Z}\left(f^{\prime}\right)_{x}\right)=f_{x}^{\prime} \mathcal{O}\left(X_{x}\right)$; thus $f^{\prime}$ changes sign at all points of $\mathscr{Z}\left(f^{\prime}\right)$.

With this at hand we can prove the

$\underset{\mathbb{Z}}{\operatorname{Proposition}}$ 2.3. $\quad$ Let $\beta \in \operatorname{Spec}_{r} \mathscr{K}$ and suppose $\operatorname{dim} \mathscr{U}_{\beta}=1$. Then $\frac{\Gamma_{\beta}}{2 \Gamma_{\beta}}$ is isomorphic to a subgroup of $\frac{\mathbb{Z}}{2 \mathbb{Z}}$. In particular, $\# F_{W_{\beta}} \leqq 2$.

Proof. First suppose that $\mathscr{Z}(f)^{\mathrm{c}} \notin \mathscr{U}_{\beta}$. Factoring $f$ as in Proposition 2.2 we can write $f=f^{\prime} \sum_{1}^{r} A_{i}^{2}$, with $\mathscr{Z}\left(f^{\prime}\right) \notin \mathscr{U}_{\beta}$. By Remark 1.2, this implies $f^{\prime}=u h^{2}$, with $u$ a unit in $U_{\beta}$. Therefore $f=u h^{2} \sum_{1}^{r} A_{i}^{2}$ so $\omega(f) \in 2 \Gamma_{\beta}$.

Let $f, g \notin 2 \Gamma_{\beta}$. In particular we have that $\mathscr{Z}(f)^{\mathrm{c}}, \mathscr{Z}(g)^{\mathrm{c}} \in \mathscr{U}_{\beta}$. Then

$$
Y=\mathscr{Z}(f)^{\mathrm{c}} \cap \mathscr{Z}(g)^{\mathrm{c}} \in \mathscr{U}_{\beta}
$$

and $f g$ does not change sign along $Y$. It follows that $\mathscr{Z}(f g)^{\mathrm{c}} \notin \mathscr{U}_{\beta}$ so that

$$
\omega(f g)=\omega(f)+\omega(g) \in 2 \Gamma_{\beta} .
$$

Thus $\frac{\Gamma_{\beta}}{2 \Gamma_{\beta}} \subset \frac{\mathbb{Z}}{2 \mathbb{Z}}$ and we are done.

Finally, it remains to see what happens when $\operatorname{dim} \mathscr{U}_{\beta}=0$. Before we recall a few facts about the orderings of $\mathscr{K}$. Let $\mathscr{S}$ denote the family of open global semianalytic subsets of $X$. This family is closed under finite unions and intersections so we can consider filters of sets of $\mathscr{S}$.

The ultrafilters of $\mathscr{S}$ are closely related to the orderings of $\mathscr{K}$. In fact, the ultrafilter theorem, cf. [Ca-An], Theorem 2.2, says that there exists a one-to-one correspondence between the orderings of $\mathscr{K}$ and the ultrafilters of $\mathscr{S}$. More precisely, if $\beta \in \operatorname{Spec}_{r} \mathscr{K}$ then $v_{\beta}=\left\{S \in \mathscr{S} \mid\left\{f_{1}>0, \ldots, f_{r}>0\right\} \subset S\right.$ for some $\left.f_{1}, \ldots, f_{r} \in \beta\right\}$ is an ultrafilter of $\mathscr{S}$. Conversely, if $v$ is an ultrafilter of $\mathscr{S}$ then $\beta=\left\{\frac{f}{g} \mid f, g \in \mathcal{O}(X)\right.$ and $\left.\{f g>0\} \in v\right\}$ is an
ordering of $\mathscr{K}$.

We remember also the tilde map which assigns to any global semianalytic set $S \subset X$ the constructible set $\tilde{S}$ of $\operatorname{Spec}_{r} \mathscr{K}$ defined by the same formula. Although the tilde map is not bijective, two global semianalytic sets $S$ and $T$ have the same tilde image, that is, $\tilde{S}=\tilde{T}$, if and only if they are generically equal, cf. [Ca-An], Proposition 2.4.

Proposition 2.4. Let $\beta_{1}, \ldots, \beta_{8} \in \operatorname{Spec}_{r} \mathscr{K}$ and suppose that $\mathscr{U}=\mathscr{U}_{\beta_{1}}=\cdots=\mathscr{U}_{\beta_{8}}$ and $\operatorname{dim} \mathscr{U}=0$. Then they are not a fan.

In particular, for any $\beta \in \operatorname{Spec}_{r} \mathscr{K}, \# F_{W_{\beta}} \leqq 4$. 
Proof. Let $v_{\beta_{1}}, \ldots, v_{\beta_{8}}$ be the maximal filters of $\mathscr{S}$ corresponding to $\beta_{1}, \ldots, \beta_{8}$. Since $\beta_{i} \neq \beta_{j}$, for $i \neq j$, there are basic open sets $B_{i} \in v_{\beta_{i}}$ such that $B_{i} \cap B_{j}=\emptyset$, for $i \neq j$.

Notice that we can reduce to a multilocal problem: If $D \in \mathscr{U}$ is a discrete set then for each $p \in D$ take a neighborhood $U\left(p, \varepsilon_{p}\right)$ of $p$ of arbitrarily small radius $\varepsilon_{p}$. Let $h_{\varepsilon} \in \mathcal{O}(X)$ be such that

$$
\bigcup_{p \in D} U\left(p, \frac{\varepsilon_{p}}{2}\right) \subset\left\{h_{\varepsilon}>0\right\} \quad \text { and } \quad X \backslash \bigcup_{p \in D} U\left(p, \varepsilon_{p}\right) \subset\left\{h_{\varepsilon}<0\right\} .
$$

Such a $h_{\varepsilon}$ can be constructed by approximating a $C^{\infty}(X)$ function with the same property, cf. $[\mathrm{H}]$, Chapter 2. By Lemma 1.1, $h_{\varepsilon} \in \beta_{i}$, for all $i$, since all these orderings have the same attached ultrafilter $\mathscr{U}$. In particular, $B_{i}^{\prime}:=\left\{h_{\varepsilon}>0\right\} \cap B_{i} \in v_{\beta_{i}}$, for any $i$ and any $\varepsilon$, and replacing $B_{i}$ by $B_{i}^{\prime}$ we may consider the $B_{i}$ 's in an arbitrarily small ball of the discrete set $D$.

This also shows that $D_{i}:=\overline{B_{i}} \cap D \in \mathscr{U}$. Therefore replacing, if necessary, $D$ by $D^{\prime}:=\bigcap_{i=1}^{8} D_{i}$ we can suppose that all the $B_{i}$ 's are adherent to the discrete set $D$, that is, $\overline{B_{i}} \cap D=D$.

Now, for any disjoint sets $B_{1} \in v_{\beta_{1}}$ and $B_{i} \in v_{\beta_{i}}, \operatorname{dim}\left(\bar{B}_{1} \cap \bar{B}_{i}\right)_{p}=0$ or 1 for each $p \in D$. Thus, we have $D=\left\{p \in D \mid \operatorname{dim}\left(\bar{B}_{1} \cap \bar{B}_{i}\right)_{p}=0\right\} \cup\left\{p \in D \mid \operatorname{dim}\left(\bar{B}_{1} \cap \bar{B}_{i}\right)_{p}=1\right\} \in \mathscr{U}$ and as $\mathscr{U}$ is an ultrafilter one and only one of these two sets is in $\mathscr{U}$. Hence we distinguish two cases:

Case 1. For any $i=2, \ldots, 8$, there exist pairwise disjoint basic open sets $B_{i} \in v_{\beta_{i}}$ such that the discrete set $D_{i}^{\prime}:=\left\{p \in D \mid \operatorname{dim}\left(\bar{B}_{1} \cap \bar{B}_{i}\right)_{p}=0\right\}$ is in $\mathscr{U}$. In this case we change $D$ by $\bigcap_{2}^{8} D_{i}^{\prime} \in \mathscr{U}$.

Let $p \in D$ and let $B_{1, p}, \ldots, B_{8, p}$ be the basic open semianalytic set germs of $B_{1}, \ldots, B_{8}$ at $p$. Since $t\left(X_{p}\right)=2$, cf. [DC2], we can write $B_{2, p} \cup \cdots \cup B_{8, p}=C_{1, p} \cup C_{2, p}$, for some basic open set germs $C_{1, p}$ and $C_{2, p}$. Now take $h_{i, p} \in \mathcal{O}\left(X_{p}\right)$ separating $\overline{B_{1, p}}$ and $\overline{C_{i, p}}$, i.e., $\overline{B_{1, p}} \subset\left\{h_{1, p}>0\right\} \cup\{p\}$ and $\overline{C_{i, p}} \subset\left\{h_{1, p}<0\right\} \cup\{p\}$. Such a function exists since $\overline{B_{1, p}}$ and $C_{i, p}$ are basic of dimension 2, cf. [Brö], Proposition 8.9 for the semialgebraic case and [DC1] for semianalytic set germs.

Hence we have $\overline{B_{1, p}} \subset\left\{h_{1, p}>0, h_{2, p}>0\right\} \cup\{p\}$ and

$$
\overline{B_{2, p}} \cup \cdots \cup \overline{B_{8, p}}=\overline{C_{1, p}} \cup \overline{C_{2, p}} \subset\left\{h_{1, p}<0\right\} \cup\left\{h_{2, p}<0\right\} \cup\{p\} .
$$

Now, by $[\mathrm{Ca}-\mathrm{An}]$, Lemma 4.3 , there exists $l_{p} \in \mathbb{N}$ such that if $h_{i, p}^{\prime}=h_{i, p} \bmod \mathrm{m}_{p}^{l_{p}}\left(\right.$ where $\mathrm{m}_{p}$ is the maximal ideal of $\left.\mathcal{O}\left(X_{p}\right)\right)$ then $\overline{B_{1, p}} \subset\left\{h_{1, p}^{\prime}>0, h_{2, p}^{\prime}>0\right\} \cup\{p\}$ and

$$
\overline{B_{2, p}} \cup \cdots \cup \overline{B_{8, p}} \subset\left\{h_{1, p}^{\prime}<0\right\} \cup\left\{h_{2, p}^{\prime}<0\right\} \cup\{p\} .
$$

Now we use Cartan's Theorem B to produce global analytic functions $H_{1}$ and $H_{2}$ such that at each $p \in D$ their germs coincide with $h_{1, p}$ and $h_{2, p}$, respectively, till order $l_{p}$ (see 
[Ca-An], Proposition 3.6.d). Hence it follows that $H_{1}$ and $H_{2}$ separate locally $B_{1, p}$ from $B_{2, p}, \ldots, B_{8, p}$ at each $p \in D$. Replacing, if necessary, each $B_{i}$ by $U \cap B_{i}$ for some open neigborhood $U$ of $D$ small enough we can conclude that $H_{1}$ and $H_{2}$ separate globally $B_{1}$ from $B_{2}, \ldots, B_{8}$ and, therefore, $\beta_{1}$ from $\left\{\beta_{2}, \ldots, \beta_{8}\right\}$. This shows that $\beta_{1}, \ldots, \beta_{8}$ are not a fan.

Case 2. There exist some $i \in\{2, \ldots, 8\}$ such that for all disjoint sets $B_{1} \in v_{\beta_{1}}$ and $B_{i} \in v_{\beta_{i}}$ the set $D^{\prime}:=\left\{p \in D \mid \operatorname{dim}\left(\bar{B}_{1} \cap \bar{B}_{i}\right)_{p}=1\right\}$ is in $\mathscr{U}$. In this case we shall suppose $i=2$ and change $D$ by $D^{\prime}$.

Assume now $U$ is an open global semianalytic neighborhood of $\left(\overline{B_{1}} \cap \overline{B_{2}}\right) \backslash D$. We claim that $U \in v_{\beta_{1}}$. For otherwise $U^{\prime}:=\operatorname{Int}(X \backslash U) \in v_{\beta_{1}}$, since $v_{\beta_{1}}$ is an ultrafilter, and defining $B_{1}^{\prime}:=B_{1} \cap U^{\prime} \in v_{\beta_{1}}$ we will have $\operatorname{dim} \overline{B_{1}^{\prime}} \cap \overline{B_{2}}=0$, against the hypothesis. Symmetrically $U \in v_{\beta_{2}}$.

Let us denote $\gamma_{p}^{1}, \ldots, \gamma_{p}^{r_{p}}$ the half-branches of $\left(\overline{B_{1}} \cap \overline{B_{2}}\right)_{p}$. Clearly

$$
\left(\gamma_{p}^{1} \cup \cdots \cup \gamma_{p}^{r_{p}}\right) \cap\left(\bar{B}_{3} \cup \cdots \cup \bar{B}_{8}\right)_{p}=\{p\}
$$

so using again Cartan's Theorem B we can find a global analytic function $f$ whose germ at each $p \in D$ is positive on $\left(\gamma_{p}^{1} \cup \cdots \cup \gamma_{p}^{r_{p}}\right)$ and negative on $\left(\bar{B}_{3} \cup \cdots \cup \bar{B}_{8}\right)_{p}$ except for $p$. Then $\{f>0\} \in v_{\beta_{1}} \cap v_{\beta_{2}}$ but $\{f<0\} \in v_{\beta_{3}} \cap \cdots \cap v_{\beta_{8}}$ which is impossible for 8-element fans.

Once computed the number of elements of the fans $F_{W_{\beta}}$ we can determine the generic invariants $s$ and $t$.

Theorem 2.5. Let $X$ be a paracompact real connected analytic manifold of dimension 2 and let $\mathscr{K}$ be its field of meromorphic functions. Then $s(\mathscr{K})=2$ and $t(\mathscr{K})=2$.

Proof. By the stability formula we have to show that for any fan $F \subset \operatorname{Spec}_{r} \mathscr{K}$, $\# F \leqq 4$. Since $F \subset F_{W_{\beta}} \cup F_{W_{\beta^{\prime}}}$ for some $\beta, \beta^{\prime} \in F$, by the preceding propositions we have to see that if $\# F_{W_{\beta}}=\# F_{W_{\beta^{\prime}}}=4$ then $F_{W_{\beta}} \cup F_{W_{\beta^{\prime}}}$ is not a fan (unless $F_{W_{\beta}}=F_{W_{\beta^{\prime}}}$ ). We can suppose that $\operatorname{dim} \mathscr{U}_{\beta}=\operatorname{dim} \mathscr{U}_{\beta^{\prime}}=0$.

If the ultrafilters $\mathscr{U}_{\beta}$ and $\mathscr{U}_{\beta^{\prime}}$ coincide then by Proposition $2.4, F_{W_{\beta}} \cup F_{W_{\beta^{\prime}}}$ is not a fan. If, on the other hand, $\mathscr{U}_{\beta} \neq \mathscr{U}_{\beta^{\prime}}$ then there are discrete disjoint sets $D \subset \mathscr{U}_{\beta}$ and $D^{\prime} \subset \mathscr{U}_{\beta^{\prime}}$. Now, let $F_{W_{\beta}}=\left\{\beta=\beta_{1}, \ldots, \beta_{4}\right\}$ and denote by $v_{\beta_{i}}$ the corresponding ultrafilters of $\mathscr{S}$. Then, similarly to Proposition 2.4 , there exist basic open sets $B_{i} \in v_{\beta_{i}}$ and function germs $f_{p}$ such that (possibly after taking a smaller $D \in \mathscr{U}_{\beta}$ and renumerating the elements of $F_{W_{\beta}}$ ) any function germ $g_{p}$ equal to $f_{p}$ up to order sufficiently high separates $B_{1, p} \cup B_{2, p}$ from $B_{3, p} \cup B_{4, p}, \forall p \in D$. Defining $f_{p}=-1$ for $p \in D^{\prime}$ and using Cartan's Theorem B we find a global analytic function which separates two orderings from the other six showing that $F_{W_{\beta}} \cup F_{W_{\beta^{\prime}}}$ is not a fan.

Concerning the invariant $t$, as $\operatorname{Spec}_{r} \mathscr{K}$ is a space of orderings with stability index 2, Corollary IV.7.9.a) of [An-Brö-Rz2] says that $t(\mathscr{K})=2$.

After this, using Proposition 2.2 we get the 
Theorem 2.6. Let $X$ be a paracompact real connected analytic manifold of dimension 2. Then $s(X)=2$, that is every basic open subset of $X$ can be written with two inequalities.

Proof. Let $B=\left\{f_{1}>0, \ldots, f_{r}>0\right\}$ be a basic open set and consider its image $\tilde{B}$ under the tilde map. By the previous theorem, $\tilde{B}=\left\{\beta \in \operatorname{Spec}_{r} \mathscr{K} \mid f>_{\beta} 0, g>_{\beta} 0\right\}$ for some $f, g \in \mathcal{O}(X)$, so defining $B^{\prime}:=\{x \in X \mid f(x)>0, g(x)>0\}, B$ and $B^{\prime}$ verify $\tilde{B}=\widetilde{B}^{\prime}$, therefore they are generically equal, that is, they differ in something of codimension at least 1 . We can suppose that $f$ and $g$ verify properties a) and b) of Proposition 2.2 since otherwise we change $f$ and $g$ by the functions $f^{\prime}$ and $g^{\prime}$ associated by that proposition. A straightforward computation checks that $B \subset B^{\prime}$. Now, if $B^{\prime} \subset B$ the result follows. Otherwise, $B^{\prime} \backslash B$ will be contained in a global analytic set of dimension 1, so we will have, $\bar{B} \backslash B^{z}=\{r=0\}$ for some non-negative $r \in \mathcal{O}(X)$. As $B^{\prime} \backslash B \subset \operatorname{Bd} B$ we have $\{r=0\} \subset \overline{\mathrm{Bd} B}^{z}$, but a basic open set does not intersect its Zariski boundary, cf. [Brö], Proposition 2.2, so $\{r=0\} \cap B=\emptyset$. Now, it is immediate to check that $B=\{f r>0, g>0\}$ and we are done.

\section{Hörmander-Lojasiewicz inequality}

The following is a generalization of a result stated by Bochnak and Risler, cf. [Bo-Rs], for analytic manifolds. We will say that a germ $\xi_{p} \in \mathcal{O}\left(X_{p}\right)$ is elliptic if $\xi_{p}$ does not change sign and its zero set is an isolated point, namely $p$.

Proposition 3.1. Let $X \subset \mathbb{R}^{n}$ be a coherent analytic set and let $D$ be a discrete subset of $X$. We fix for each $p \in D$ a non-negative analytic germ $\rho_{p} \in \mathcal{O}\left(X_{p}\right)$ such that $\mathscr{Z}\left(\rho_{p}\right)=\{p\}$. Then there exists an analytic function $\xi \in \mathcal{O}(X)$ which is a sum of squares of meromorphic functions over $X$ such that $\mathscr{Z}(\xi)=D$ and $\xi_{p} \mathcal{O}\left(X_{p}\right)=\rho_{p} \mathcal{O}\left(X_{p}\right), \forall p \in D$.

Proof. If $X$ is an analytic manifold, i.e. smooth, it is known, cf. [Bo-Rs], Lemma 1, that there exists $\xi \in \mathcal{O}(X)$ such that $\mathscr{Z}(\xi)=D$ and $\forall p \in D, \xi_{p} \mathcal{O}\left(X_{p}\right)=\rho_{p} \mathcal{O}\left(X_{p}\right)$. In these conditions $\xi$ is a sum of squares of meromorphic functions, cf. [Bo-Ku-Sh], Theorem 1.

In the general case, as $\rho_{p} \in \mathcal{O}\left(X_{p}\right)$ is non-negative then $\rho_{p} h_{p}^{2}=g_{1, p}^{2}+\cdots+g_{s, p}^{2}$, for some $h_{p}, g_{i, p} \in \mathcal{O}\left(X_{p}\right)$ and $\mathscr{Z}\left(h_{p}\right) \subset \mathscr{Z}\left(\rho_{p}\right)$, cf. [An-Brö-Rz2], Proposition VIII.2.8. Let us define $c_{p}:=\rho_{p} h_{p}^{2}$ and let $g_{1, p}^{\prime}, \ldots, g_{s, p}^{\prime} \in \mathcal{O}\left(\mathbb{R}_{p}^{n}\right)$ be representatives of

$$
g_{1, p}, \ldots, g_{s, p} \in \mathcal{O}\left(X_{p}\right)=\frac{\mathcal{O}\left(\mathbb{R}_{p}^{n}\right)}{\mathscr{J}\left(X_{p}\right)} .
$$

If $r \in \mathcal{O}\left(\mathbb{R}_{p}^{n}\right)$ is a positive equation of $X_{p}$ then $c_{p}^{\prime}=r+g_{1, p}^{\prime 2}+\cdots+g_{s, p}^{\prime 2}$ is a representative of $c_{p}$ which is elliptic as a germ in $\mathcal{O}\left(\mathbb{R}_{p}^{n}\right)$. In the same way we can take a representative of $h_{p}^{2}$, which will be denoted as $b_{p}^{\prime}$, elliptic in $\mathcal{O}\left(\mathbb{R}_{p}^{n}\right)\left(\right.$ remember that $\left.\mathscr{Z}\left(h_{p}\right) \subset \mathscr{Z}\left(\rho_{p}\right)\right)$.

Now, we can apply the result for analytic manifolds to find a sum of squares of meromorphic functions $\xi^{\prime \prime} \in \mathcal{O}\left(\mathbb{R}^{n}\right)$ such that $\mathscr{Z}\left(\xi^{\prime \prime}\right)=D$ and $\xi_{p}^{\prime \prime} \mathcal{O}\left(\mathbb{R}_{p}^{n}\right)=c_{p}^{\prime} \mathcal{O}\left(\mathbb{R}_{p}^{n}\right), \forall p \in D$. Analogously, there exists $H \in \mathcal{O}\left(\mathbb{R}^{n}\right)$ which is also a sum of squares of meromorphic functions such that $\mathscr{Z}(H)=D$ and $H_{p} \mathcal{O}\left(\mathbb{R}_{p}^{n}\right)=b_{p}^{\prime} \mathcal{O}\left(\mathbb{R}_{p}^{n}\right), \forall p \in D$. Let $\xi_{1}$ and $h_{1}$ denote the classes of $\xi^{\prime \prime}$ and $H$ in $\mathcal{O}(X)=\frac{\mathcal{O}\left(\mathbb{R}^{n}\right)}{\mathscr{J}(X)}$, where $\mathscr{J}(X)=\left\{f \in \mathcal{O}\left(\mathbb{R}^{n}\right)|f|_{X}=0\right\}$. We have that 
$\xi:=\frac{\xi_{1}}{h_{1}} \in \mathscr{K}(X)$ is an analytic function in $X \backslash D$ and for each $p \in D$,

$$
\xi_{p}=\frac{\xi_{1, p}}{h_{1, p}}=u_{p} \frac{c_{p}}{h_{p}^{2}}=u_{p} \rho_{p} \in \mathcal{O}\left(X_{p}\right),
$$

where $u_{p}$ is a unit of $\mathcal{O}\left(X_{p}\right)$. Thus $\xi$ defines a global section of the sheaf $\mathcal{O}_{X}$ of analytic functions and, since $X$ is coherent, a global analytic function whence the result follows.

Now, we state the Hörmander-Lojasiewicz inequality in the following useful form (see [B-C-R], Lemma 7.7.10, for the semialgebraic case).

Proposition 3.2. Let $X$ be an analytic manifold of dimension 2 and let $T \subset X$ be a global closed semianalytic set. Given $f, g \in \mathcal{O}(X)$ there exist $p, q \in \mathcal{O}(X)$ such that the following hold:

a) $p>0, q \geqq 0$ on $X$.

b) $\operatorname{sign}(p f+q g)=\operatorname{sign} f$ over $T$.

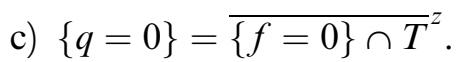

Proof. The global analytic set $Y:=\{f=0\}$ has the irredundant decomposition $Y=\left(\bigcup_{i \in I} A_{i}\right) \cup\left(\bigcup_{j \in J}\left\{x_{j}\right\}\right)$, where $I$ and $J$ are subsets of $\mathbb{Z}$, the $A_{i}$ 's are irreducible global analytic sets of dimension 1 and $\bigcup_{j \in J}\left\{x_{j}\right\}$ is a discrete set. We introduce the following definitions (see Figure 1):

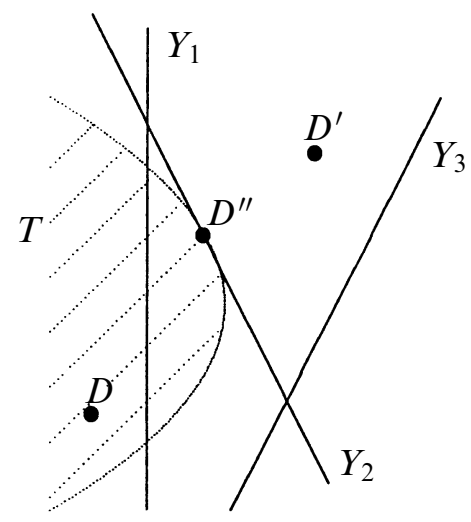

Figure 1

$$
\begin{aligned}
& Y_{1}:=\bigcup_{i \in I_{1}} A_{i}, \text { where } I_{1}:=\left\{i \in I \mid \operatorname{dim} A_{i} \cap T=1\right\} . \\
& Y_{2}:=\bigcup_{i \in I_{2}} A_{i}, \text { where } I_{2}:=\left\{i \in I \mid \operatorname{dim} A_{i} \cap T=0\right\} .
\end{aligned}
$$




$$
\begin{aligned}
& Y_{3}:=\bigcup_{i \in I_{3}} A_{i}, \text { where } I_{3}:=\left\{i \in I \mid A_{i} \cap T=\emptyset\right\} . \\
& D:=\left(\bigcup_{j \in J}\left\{x_{j}\right\}\right) \cap T, D^{\prime}:=\left(\bigcup_{j \in J}\left\{x_{j}\right\}\right) \backslash D, D^{\prime \prime}:=Y_{2} \cap T .
\end{aligned}
$$

We note that $Y_{1}, Y_{2}$ and $Y_{3}$ are global analytic sets of dimension 1 and that $D$, $D^{\prime}$ and $D^{\prime \prime}$ are discrete sets. Also notice that $Y=Y_{1} \cup Y_{2} \cup Y_{3} \cup D \cup D^{\prime}$ and that

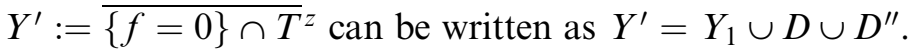

First of all, using Proposition 3.1, we factor $f$ as $f=f^{\prime} H_{D} H_{D^{\prime}}^{\prime}$, where $H_{D}, H_{D^{\prime}}^{\prime} \in \mathcal{O}(X)$ are sums of squares of meromorphic functions whose zero set are respectively $D$ and $D^{\prime}$, and $f^{\prime} \in \mathcal{O}(X)$ does not vanish at any point of $D \cup D^{\prime}$.

For each $x \in X$ we have $f_{x}^{\prime}=h_{1, x} h_{2, x} h_{3, x}$ where $\mathscr{Z}\left(h_{1, x}\right)=Y_{1, x}, \mathscr{Z}\left(h_{2, x}\right)=Y_{2, x}$ and $\mathscr{Z}\left(h_{3, x}\right)=Y_{3, x}$. The germs $h_{i, x}$ are determined up to units and, of course, if $x \notin Y_{i}$ then $h_{i, x}$ is a unit. Now, let $\mathscr{F}$ be the sheaf defined as

$$
\mathscr{F}_{x}= \begin{cases}h_{1, x}^{2} \mathcal{O}\left(X_{x}\right) & \text { if } x \in Y_{1} \\ \mathcal{O}\left(X_{x}\right) & \text { if } x \notin Y_{1}\end{cases}
$$

Since $\mathscr{F}$ is coherent there exists a finite number of global sections $g_{1}, \ldots, g_{t} \in \mathcal{O}(X)$ which generate each stalk $\mathscr{F}_{x}, x \in X$, cf. [Co]. Setting $F:=f^{\prime 2}+g_{1}^{2}+\cdots+g_{t}^{2}$, we have $\mathscr{Z}(F)=Y_{1}$. Also, the fraction $\frac{F}{f}$ is analytic on $T \backslash\left(D \cup D^{\prime \prime}\right)$ for if $x \in Y_{1} \cap T$ and $x \notin D \cup D^{\prime \prime}$ then $x \in Y_{1} \backslash\left(Y_{2} \cup Y_{3}\right)$ and therefore $f_{x}^{\prime}=h_{1, x}, g_{i, x}=a_{i, x} h_{1, x}^{2}$ for some $a_{i, x} \in \mathcal{O}\left(X_{x}\right)$ and

$$
\frac{F_{x}}{f_{x}}=\frac{h_{1, x}^{2}\left(1+a_{1, x}^{2} h_{1, x}^{2}+\cdots+a_{t, x}^{2} h_{1, x}^{2}\right)}{h_{1, x} H_{D, x} H_{D^{\prime}, x}^{\prime}} \in \mathcal{O}\left(X_{x}\right) .
$$

Suppose now that $x \in D^{\prime \prime}$. Then $Y_{x}=Y_{1, x} \cup Y_{2, x}$ so $f_{x}^{\prime}=h_{1, x} h_{2, x}$ for in this case $Y_{3, x}=\emptyset$ and $h_{3, x}$ is a unit. We apply the Hörmander-Lojasiewicz inequality for semianalytic set germs, cf. [DC-An], Proposition 1.1 , to $h_{2, x}, 1_{x}$ (the germ at $x$ of the function identically equal to 1$)$ and $T_{x}$ obtaining $p_{x}^{\prime}, q_{x}^{\prime} \in \mathcal{O}\left(X_{x}\right)$ such that $p_{x}^{\prime}>0, q_{x}^{\prime} \geqq 0$, $\operatorname{sign}\left(p_{x}^{\prime} h_{2, x}+q_{x}^{\prime}\right)=\operatorname{sign} h_{2, x}$ on $T_{x}$ and $\mathscr{Z}\left(q_{x}\right)={\overline{Y_{2, x}} \cap T_{x}}^{2}=\{x\}$. Thus the germ $q_{x}^{\prime}$ is elliptic for each $x \in D^{\prime \prime}$. Now, by Proposition 3.1 there exists a sum of squares of meromorphic functions $Q \in \mathcal{O}(X)$, such that $\mathscr{Z}(Q)=D^{\prime \prime}$ and $Q_{x}=q_{x}^{\prime} u_{x}, \forall x \in D^{\prime \prime}$, where the $u_{x}$ 's are units.

We define the function $G$ on $T$ as

$$
G= \begin{cases}\left|\frac{F H_{D} Q^{2} g}{f}\right| & \text { if } x \in T \backslash D^{\prime \prime} \\ 0 & \text { if } x \in D^{\prime \prime}\end{cases}
$$
It can be checked that $G$ is a continuous function on $T$. Indeed, if $x \in T \backslash D^{\prime \prime}$ then $\frac{F}{f}$ is
analytic and so $G$ is continuous; if $x \in D^{\prime \prime}$ then 


$$
\frac{F_{x} H_{D, x} Q_{x}^{2} g_{x}}{f_{x}}=\frac{h_{1, x}^{2}\left(1+a_{1, x}^{2} h_{1, x}^{2}+\cdots+a_{t, x}^{2} h_{1, x}^{2}\right) H_{D, x} q_{x}^{\prime 2} u_{x}^{2} g_{x}}{h_{1, x} h_{2, x} H_{D, x} H_{D^{\prime}, x}^{\prime}} .
$$

Furthermore, from the proof of the local Hörmander-Łojasiewicz inequality it follows that $\left|q_{x}^{\prime}\right| \leqq b\left|h_{2, x}\right|$ on $T_{x}$ for some constant $b$, that is, $\frac{\left|q_{x}^{\prime}\right|}{\left|h_{2, x}\right|} \leqq b$ (on $T_{x}$ ) hence the quotient above tends to 0 on $T_{x}$, from which the continuity of $G$ follows.

Now, by Tietze's theorem $G$ can be extended to a continuous function of all of $X$, that can be supposed to be non-negative, which we shall denote by $G^{\prime}$. Set $G^{\prime \prime}:=G^{\prime}+1$ and take an analytic approximation of $G^{\prime \prime}, p \in \mathcal{O}(X)$ such that $p(x)>G^{\prime}(x), \forall x \in X$, cf. [H].

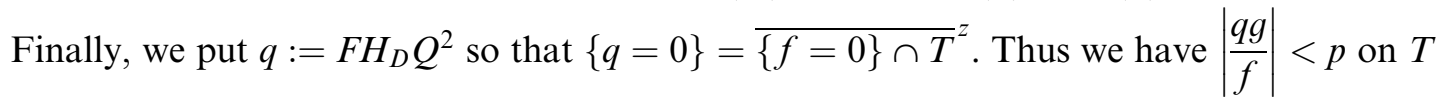
and, in particular, sign $(p f+q g)=\operatorname{sign} f$ on $T$ finishing the proof.

From this we obtain Hörmander-Łojasiewicz inequality in the form of [An-BröRz2], III.1.12. Definining $\langle f, g\rangle(x):=\operatorname{sign} f(x)+\operatorname{sign} g(x)$, where $\operatorname{sign} \in\{-1,0,1\}$, we can state

HL. Let $T \in \mathscr{C}$ and $f, g \in \mathcal{O}(X)$ such that $\{f=0\} \cap T \subset\{g=0\}$. Then there exists $f^{\prime} \in \mathcal{O}(X)$ such that:

a) $\operatorname{sign} f^{\prime}=\operatorname{sign} f$ on $T$,

b) $\operatorname{sign} f^{\prime}=\operatorname{sign} g$ on $\{f=0\}$ and

c) $\langle f, g\rangle(x)=\left\langle f^{\prime}, f^{\prime} f g\right\rangle(x), \forall x \in X$.

Proof. Take $f^{\prime}=p f+q g$ as in Proposition 3.2. Thus, we have condition a).

On the other hand, as $T \cap\{f=0\} \subset\{g=0\}$ then $\overline{T \cap\{f=0\}}^{z} \subset\{g=0\}$, that is, $\{q=0\} \subset\{g=0\}$. Thus if $g \neq 0$ then $q>0$. Now, on $\{f=0\}$ it is $f^{\prime}=q g$ so $\operatorname{sign} f^{\prime}=\operatorname{sign} g$ and we get condition $\mathrm{b}$ ).

Finally, as sign $\in\{-1,0,1\}$ it is straigthforward to check case by case that condition c) is verified.

With these tools we get the pasting lemmas similarly to [An-Brö-Rz2], Lemmas V.2.8 and V.2.13, which will allow us to pass from generic to non-generic bounds by induction.

Lemma 3.3. Let $X$ be an analytic manifold of dimension 2, $Y \subset X$ an analytic set of dimension 1 and $B \subset X$ a closed global semianalytic set. Assume that

$$
B \backslash Y=\left\{a_{1} \geqq 0, \ldots, a_{k} \geqq 0\right\} \backslash Y \quad \text { and } \quad B \cap Y=\left\{b_{1} \geqq 0, \ldots, b_{l} \geqq 0\right\} \cap Y
$$

for suitable $a_{i}, b_{i} \in \mathcal{O}(X)$. Then there exist $c_{1}, \ldots, c_{m} \in \mathcal{O}(X), m \leqq k+l$, such that $B=\left\{c_{1} \geqq 0, \ldots, c_{m} \geqq 0\right\}$. 
Lemma 3.4. Let $X$ be an analytic manifold of dimension 2 and $S \subset X$ an open global semianalytic set. If $Y \subset X$ is an analytic set of dimension 1 and $B_{1}, \ldots, B_{l}$ are basic open sets such that

$$
S \backslash Y=\left(B_{1} \cup \cdots \cup B_{m}\right) \backslash Y \quad \text { and } \quad S \cap Y=\left(B_{m+1} \cup \cdots \cup B_{l}\right) \cap Y
$$

then $S=B_{1}^{\prime} \cup \cdots \cup B_{l}^{\prime}$ for some basic open sets $B_{i}^{\prime} \subset X$.

As a consequence we also get the

Finiteness Theorem. Let $X$ be an analytic manifold of dimension 2 and let $S \subset X$ be an open (resp. closed) global semianalytic set. Then $S$ can be written as a union of basic open (resp. closed) sets.

Proof. We do the open case. The closed one follows by complementation. Now, $S$ is generically equal to a union of basic open sets, i.e.

$$
S \backslash Y=\left(B_{1} \cup \cdots \cup B_{m}\right) \backslash Y
$$

and $S \cap Y$ is open in $Y$. By Theorem 4.4 below $S \cap Y=\{f>0\} \cap Y$ so that by Lemma 3.4, $S=B_{1}^{\prime} \cup \cdots \cup B_{m}^{\prime} \cup B_{m+1}^{\prime}$.

\section{Computation of the complexity invariants}

In order to use the above pasting lemmas we need to know the invariants $s, \bar{s}, t$ and $\bar{t}$ of analytic sets of dimension 1.

We recall that every irreducible complex analytic set has an essentially unique normalization, see [Gr-Rm], Chapter VIII and [Nar], Chapter VI, and that in the real case it is also possible to define the normalization in a satisfactory way (at least for a coherent analytic set) and compatible with the normalization of its complexification, cf. [G-M-T], Chapter IV.3.

Theorem 4.1. Let $Y \subset \mathbb{R}^{n}$ be an irreducible analytic set of dimension 1 and let $\pi: Y^{\prime} \rightarrow Y$ be its normalization. Then the quotient fields of $\mathcal{O}(Y)$ and $\mathcal{O}\left(Y^{\prime}\right)$, denoted respectively by $\mathscr{K}(Y)$ and $\mathscr{K}\left(Y^{\prime}\right)$, are isomorphic.

Proof. The normalization $\pi: Y^{\prime} \rightarrow Y$ induces an injective map $\pi^{*}: \mathcal{O}(Y) \hookrightarrow \mathcal{O}\left(Y^{\prime}\right)$ that can be extended to $\pi^{*}: \mathscr{K}(Y) \hookrightarrow \mathscr{K}\left(Y^{\prime}\right)$. We are to see that $\pi^{*}$ is the isomorphism we seek for.

First, we have that $\pi: Y^{\prime} \backslash \pi^{-1}(D) \rightarrow Y \backslash D$ is an analytic isomorphism for some discrete subset $D \subset Y$. Thus, for $f^{\prime} \in \mathcal{O}\left(Y^{\prime}\right)$ we can define $f \in \mathcal{O}(Y \backslash D)$ by composition as $f=f^{\prime} \circ\left(\left.\pi\right|_{Y^{\prime} \backslash \pi^{-1}(D)}\right)^{-1}$. Now, let $p \in D$ and let $Y_{p}=Y_{1, p} \cup \cdots \cup Y_{r, p}$ be the decomposition of $Y_{p}$ in irreducible analytic set germs. As $\pi$ is a surjective map in dimension 1 then there exists a neigborhood $U$ of $p$ such that $\pi^{-1}(U)=W_{1} \cup \cdots \cup W_{r}$, cf. [Gr-Rm], Theorem VIII.1.2, and the restriction of $\pi$ to each $W_{i}$ induces the normalization of $Y_{i, p}$. The total quotient ring of $\mathcal{O}\left(Y_{p}\right)$, denoted as $\mathscr{K}\left(Y_{p}\right)$, is isomorphic to the direct product $\prod \mathscr{K}\left(Y_{i, p}\right)$ 
and thus the restrictions of $f^{\prime}$ to each $W_{i}$ defines an element of $\mathscr{K}\left(Y_{p}\right)$. Therefore, the germ $f_{p} \in \mathscr{K}\left(Y_{p}\right)$ is well defined and then can be written as $f_{p}=\frac{g_{p}}{h_{p}}$, with $g_{p}, h_{p} \in \mathcal{O}\left(Y_{p}\right)$.

In this way we obtain a global section in the sheaf of meromorphic functions of $Y$ and, being $Y$ a coherent analytic set, it defines a meromorphic function. In conclusion, the map $\pi^{*}$ is bijective and therefore an isomorphism from $\mathscr{K}(Y)$ to $\mathscr{K}\left(Y^{\prime}\right)$.

In particular, this isomorphism shows that the Artin-Lang property is valid for irreducible one-dimensional analytic sets for it is valid in the case of an analytic manifold of dimension 1, cf. [An-Be]. Moreover, we have the following

Theorem 4.2. Let $Y \subset \mathbb{R}^{n}$ be an irreducible analytic set of dimension 1. Then $s(\mathscr{K}(Y))=t(\mathscr{K}(Y))=1$.

Proposition 4.3. Let $Y \subset \mathbb{R}^{n}$ be a global irreducible analytic set of dimension 1 .

a) Any open semianalytic set is basic global and $s(Y)=1$.

b) Any closed semianalytic set is basic global and $\bar{s}(Y)=1$.

Proof. a) Let $B$ be a semianalytic open set. Then it is global, cf. [Ca-An], Lemma 3.1 , and by the previous theorem it is generically equal to $B^{\prime}=\{f>0\}$, for some $f \in \mathcal{O}(Y)$. Thus, $D:=B^{\prime} \backslash B$ is a discrete set and multiplying $f$ by a non-negative function vanishing at $D$ we can suppose $B^{\prime} \subset B$.

Now, $D^{\prime}:=B \backslash B^{\prime}$ is a discrete set and each $p \in D^{\prime}$ is in the interior of $\overline{B^{\prime}}$, that is, $f_{p}$ is elliptic at each point of $D^{\prime}$. By Proposition 3.1 there exists $f^{\prime} \in \mathcal{O}(Y)$ such that $\mathscr{Z}\left(f^{\prime}\right)=D^{\prime}$ and $f_{p}^{\prime} \mathcal{O}\left(Y_{p}\right)=f_{p} \mathcal{O}\left(Y_{p}\right), \forall p \in D^{\prime}$. Thus, $f^{\prime \prime}=\frac{f}{f^{\prime}} \in \mathcal{O}(Y)$ and $B=\left\{f^{\prime \prime}>0\right\}$.

b) For closed sets the result follows by complementation.

For the applications we need the values of $s(Y), \bar{s}(Y), t(Y)$ and $\bar{t}(Y)$ when $Y$ is nonirreducible. We still have:

Theorem 4.4. Let $Y \subset \mathbb{R}^{n}$ be an analytic set of pure dimension 1 , possibly reducible. Then $s(Y)=\bar{s}(Y)=t(Y)=\bar{t}(Y)=1$.

Proof. We can suppose $Y=\bigcup_{1}^{\infty} Y_{i}$, where each $Y_{i}$ is an irreducible analytic set of dimension 1 and the family $\left\{Y_{i}\right\}_{1}^{\infty}$ is locally finite. Let $r_{i} \in \mathcal{O}\left(\mathbb{R}^{n}\right)$ denote a positive equation of $\overline{Y \backslash Y_{i}}$ and let $B=\left\{g_{1}>0, \ldots, g_{s}>0\right\} \cap Y$, with $g_{i} \in \mathcal{O}\left(\mathbb{R}^{n}\right)$, a basic open set of $Y$. By Proposition 4.3, $B_{i}:=B \cap Y_{i}$ can be written as $B_{i}=\left\{h_{i}>0\right\} \cap Y_{i}$ for suitable $h_{i} \in \mathcal{O}\left(\mathbb{R}^{n}\right)$. Now, the function germs $f_{x}:=\sum_{i \in J_{x}} h_{i, x} r_{i, x}$, with $J_{x}:=\left\{i \mid x \in Y_{i}\right\}$, define a global section of the sheaf $\mathcal{O}_{Y}$. Since $Y$ has dimension 1 it is coherent and therefore this section defines a global analytic function $f \in \mathcal{O}(Y)$. Thus, $B$ and $B^{\prime}:=\{f>0\}$ differ in a discrete set and using the same argument of Proposition 4.3 we can conclude $B=\left\{f^{\prime \prime}>0\right\} \cap Y$, so that $s(Y)=1$. 
In a similar way it can be shown that $\bar{s}=t=\bar{t}=1$.

Once known the invariants $s, \bar{s}, t$ and $\bar{t}$ in dimension 1, we can apply the pasting lemmas to compute the complexity invariants in any paracompact analytic manifold of dimension 2, following the pattern of [Brö], Theorems 7.6, 9.4 and Proposition 9.7 and pointing out that the examples [An-Brö-Rz2], VI.7.2.b) and VI.7.2.e) apply in our case.

Proposition 4.5. Let $X \subset \mathbb{R}^{n}$ be an analytic manifold of dimension 2. Then $\bar{s}(X)=3$, $\bar{t}(X)=2$ and $t(X)=3$.

\section{References}

[An-Be] C. Andradas, E. Becker, A note on the real spectrum of analytic functions on an analytic manifold of dimension one, Springer Lect. Notes Math. 1420 (1990), 1-21.

[An-Brö-Rz1] C. Andradas, L. Bröcker, J. Ruiz, Minimal generation of basic open semianalytic sets, Invent. Math. 92 (1988), 409-430.

[An-Brö-Rz2] C. Andradas, L. Bröcker, J. Ruiz, Constructible sets in real geometry, Springer-Verlag, 1996.

[Be] E. Becker, On the real spectrum of a ring and its applications to semialgebraic geometry, Bull. Amer. Math. Soc. (N.S.) 15 (1986), 19-60.

[B-C-R] J. Bochnak, M. Coste, M. F. Roy, Géométrie algébrique réelle, Springer-Verlag, 1987.

[Bo-Ku-Sh] J. Bochnak, W. Kucharz, M. Shiota, On equivalence of ideals of real global analytic functions and the 17th Hilbert problem, Invent. Math. 63 (1981), 403-421.

[Bo-Rs] J. Bochnak, J. Risler, Le théorème des zéros pour les variétés analytiques réelles de dimension 2 , Ann. Sci. Éc. Norm. Sup. 8 (1975), 353-364.

[Brö] L. Bröcker, On basic semialgebraic sets, Expo. Math. 9 (1991), 289-334.

[Ca1] A. Castilla, Artin-Lang property for analytic manifolds of dimension two, Math. Z. 217 (1994), 514.

[Ca2] A. Castilla, Sums of $2 n$-th powers of meromorphic functions with compact zero set, Springer Lect. Notes Math. 1524 (1991), 174-177.

[Ca-An] A. Castilla, C. Andradas, Connected components of global semianalytic subsets of 2-dimensional analytic manifolds, J. reine angew. Math. 475 (1996), 137-148.

[Co] S. Coen, Sul rango dei fasci coerenti, Boll. Un. Mat. Ital. 22 (1967), 373-383.

[DC1] A. Díaz-Cano, Índice de estabilidad y descripción de conjuntos semianalíticos, Ph.D. Thesis, Universidad Complutense de Madrid, 1999.

[DC2] A. Díaz-Cano, The $t$-invariant of analytic set germs of dimension 2, J. Pure Appl. Algebra, to appear.

[DC-An] A. Díaz-Cano, C. Andradas, Stability index of closed semianalytic set germs, Math. Z. 229 (1998), 743-751.

[Gr-Rm] H. Grauert, R. Remmert, Coherent Analytic Sheaves, Springer Grundl. math. Wiss. 265 (1984).

[G-M-T] F. Guaraldo, P. Macri, A. Tancredi, Topics on Real Analytic Spaces, Adv. Lect. Math., Vieweg, 1986.

[H] M. Hirsch, Differential Topology, Springer-Verlag, 1976.

[Jw] P. Jaworski, The 17-th Hilbert problem for noncompact real analytic manifolds, Springer Lect. Notes Math. 1524 (1991), 289-295.

[Ma] M. Marshall, Spaces of orderings and abstract real spectra, Springer Lect. Notes Math. 1636 (1996).

[Nar] R. Narasimhan, Introduction to the theory of analytic spaces, Springer-Verlag, 1966.

[Rz] J. Ruiz, A note on a separation problem, Arch. Math. 43 (1984), 422-426.

[Sch] C. Scheiderer, Stability index of real varieties, Invent. Math. 97 (1989), 467-483.

Departamento de Álgebra, Facultad de Matemáticas, Universidad Complutense de Madrid, 28040 Madrid, Spain e-mail: Carlos_Andradas@mat.ucm.es e-mail: Antonio_DiazCano@mat.ucm.es 